\title{
AN AGENT BASED SIMULATION MODEL TO LOCATING SELLERS USING GEOGRAPHIC INFORMATION SYSTEMS
}

\author{
Ali A. Pouyan, School of Computer and IT Engineering, Shahrood University of Technology, Shahrood, Iran, \\ apouyan@shahroodut.ac.ir \\ Ali Bazghandi, School of Computer and IT Engineering, Shahrood University of Technology, Shahrood, Iran, \\ bazghandi@shahroodut.ac.ir
}

\begin{abstract}
In this paper, a generic model for locating sellers in a geographical area is presented. The proposed model is based on buyers' side attributes including price, quality, distance, income and discount, which determine the location of the prospective sellers. The required information for the model is acquired from buyers and the geographic information system (GIS). It is assumed that the agents (sellers and buyers) transact in a multi agent system (MAS) environment. Buyer agents act according to the adjacency to market place, incoming level, quality of commodity, and buying mentality background. The advantage of the proposed model is that it combines agents' behavior with GIS. While GIS enables us to model geographic space, MAS models the behavior of intelligent agents within a specific geographical space.
\end{abstract}

Keywords: Model, Buyer-Seller, Multi Agent System, GIS, Simulation

\section{INTRODUCTION}

We investigate sellers' sale in some locations in a dynamic, decentralized, open, uncertain environment. Sellers, who are similar in most of their attributes, sell products. Buyers consider goods price and has certain expectations of distance, quality and the trusty of a seller, which reflects the seller's ability to provide the product at the buyer's expectation level. The seller's offered price can be compared to its competitors in the market. The buyer wants to purchase some products from a seller who meets its expectations of quality and service and purchase it at the lowest possible price (from nearest place) in the market. Number of products, which he buys, depends on his income level. Income level differs from one buyer to another. We use one number to show each of the three buyer groups: lower class, middle class, higher class. The buyer considers distance from sellers, too. At the same time the buyer wants to reduce its chances of interacting with dishonest, poor quality, far away sellers. Sellers and buyers can enter and leave the market anytime. Players in the market need not exhibit the same behavior all the time; Buyers can gauge the quality of the product after actually receiving the product. Multiple transactions can happen between seller and buyer. There is no limitation on the number of sellers and buyers in the market, but in our approach the number of sellers is limited and location of sellers and seller category are determined by user. We try to approximate model circumstances to traditional commerce market.

At the first time, buyer agents entering the shop don't know seller is honest or not, is the seller being lying or not, is the lowest priced proposed by the seller, and if it is the best choice or not. Is transaction with the nearest seller beneficial?

Agents have been equipped with information which enables them to make the most rational decision based on all the information that they have gathered. They should be able to learn from their past experiences.

In recent research and studies the development of Multi Agent Geo-simulations has been widely focused [5]. However, the agents have not been considered autonomous [1]. Furthermore, the agents do not have learning abilities [5]. In most studies [2], [7] simulations happen in certain and trusted market places because of the nature of the problem. Jiang [3] explores the possibilities of the agent-based approach through some practical examples from urban and environmental systems. Some research works describe multi agent system (MAS) in geographic information systems (GIS) quite theoretically [4]. Certain researchers have not included GIS parameters in their works [6], [9].

In our recent research [10] we have considered GIS parameters in seller/buyer environment as a system. In this work we propose a flexible model for shopping that buyers consider proximity to shops and sellers' reputation. At last sellers' sales are compared and sellers with highest gain are considered.

The remainder of the paper is organized as follows: section 2 introduces the agent base simulation; 
section 3 presents GIS based modeling. In section 4, we explain our proposed methodology. Section 5, provides a case study, and finally section 6 draws the conclusions.

\section{AGENT BASED SIMULATION MODELING}

A model is a simplified representation of reality (i.e. of one or more processes that are believed to occur in the real-world; [11], of which there are several types. A model can be constructed as a computer program that uses (usually to some degree) a simplified digital representation of one or more aspects of the realworld, transforming them to create a new representation.

There is no universal agreement on the precise definition of the term "agent", although definitions tend to agree on more points that they don't agree constituent parts [12].

The concept of an agent is meant to be a tool for analyzing a system, not an absolute classification where entities can be defined as agents or non-agents [13]. For example, some modelers consider any type of independent component (i.e. software, model, individual, etc), to be an agent.

Agent based modelling is not the same as objectoriented simulation, although the object-oriented paradigm provides a suitable medium for the development of agent-based models. For this reason, agent based modelling systems are invariably objectoriented [8].

Agent-based models are comprised of multiple, interacting agents situated within a model or simulation environment. A relationship between agents is specified, linking agents to other agents and /or other entities within a system. Relationships may be specified in a variety of ways, from simply reactive (i.e. agents only perform actions when triggered to do so by some external stimulus e.g. actions of another agent), to goal-directed (i.e. seeking a particular goal). The behavior of agents can be scheduled to take place synchronously (i.e. every agent performs actions at each discrete time step), or asynchronously (i.e. agent actions are scheduled by the actions of other agents, and/or with reference to a clock).

In the modeling context, agent-based models can be used as experimental media for running and observing agent-based simulations. The ability to simulate individual actions of many diverse agents and measure the resulting system behavior and outcomes over time (e.g. changes in patterns of pedestrian emergency egress), means agent-based models can be useful tools for studying the effects on processes. They operate at multiple scales and organizational levels, and their effects [14]. In particular, the roots of agent based modelling are within the simulation of human social behavior and individual decision-making [12].

\section{GIS BASED MODELLING}

Essentially, geospatial models depend on the location of the features or phenomena being modeled, such that if one or more of those locations change, the results of the model change [15].

GIS's are particularly useful medium for representing model input and output of a geospatial nature. However, GIS's are not well suited to dynamic modeling [16]. There are several good reasons to justify why the use, or linkage of GIS with simulation / modeling systems, is an effective means of modeling when spatial and temporal analysis is necessary.

Current commercial and public domain GIS software systems all contain numerous tools for acquiring, preprocessing, and transforming data. Their use in modelling includes data management, format conversion, projection change, re-sampling, rastervector conversion, etc. GIS also include excellent tools for visualization / mapping, rendering, querying, and analyzing model results, as well as assessing the accuracies and uncertainties associated with inputs and outputs.

\section{THE PROPOSED METHODOLOGY}

In the proposed method, buyers are distributed in decentralized area. Openness, dynamism, uncertainness and untrusted shops are our model attributes. Buyer agents with spatial dimensions model the reputation and accessibility (proximity) of dynamic sellers based on their direct interactions with them. In some research works the model is based on the sellers' reputation only [9]. The goal of this paper is to apply a learning strategy for buyers to compute sellers' reputation and accessibility. A buyer has certain expectations of good quality, reputation of a seller and seller's adjacency in GIS environment to reflect the seller's ability to provide the product at the buyer's expectation level, and its price compared to its competitors in the market.

The buyers' goal is to purchase from a seller who will maximize its valuation of the product, which is a 
function of the price, quality of the product and adjacency of salesroom. At the same time it is desired to avoid interaction with dishonest, poor quality and far sellers in the market. The reputation of the seller is used to weed out dishonest or poor quality sellers. Far sellers with low quality omit from dealing list. In this paper we use the following notation: Subscript represents the agent computing the rating. Superscript represents the agent about whom the rating is being computed. The information in the parenthesis in the superscript is the kind of rating being computed. For example, every time buyer $b$ purchases a product from the seller, it computes an evaluator function $\mathrm{D}_{\mathrm{s}}^{\mathrm{b}}$ of the seller $s$ by buyer $b$. The evaluator function of seller " $s$ " by buyer " $b$ " is computed as shown in equation 1 .

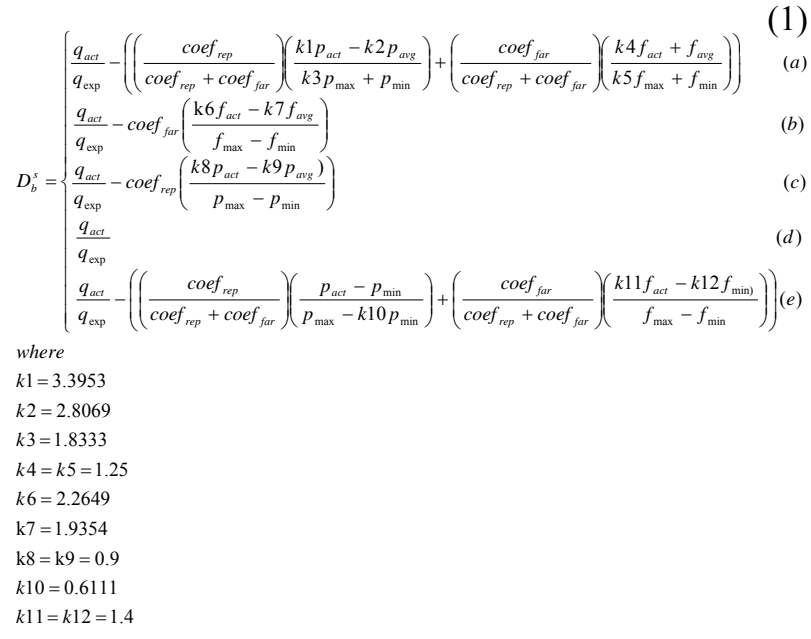

where (a), (b), (c), (d), (e) are conditions comes below:

(a) : if $\mathrm{q}_{\mathrm{act}} \geq \mathrm{q}_{\min }$ and $\mathrm{p}_{\mathrm{act}}>\mathrm{p}_{\mathrm{avg}}$ and $\mathrm{f}_{\mathrm{act}}>\mathrm{f}_{\mathrm{avg}}$

(b) : if $\mathrm{q}_{\mathrm{act}} \geq \mathrm{q}_{\min }$ and $\mathrm{p}_{\text {act }}<\mathrm{p}_{\text {avg }}$ and $\mathrm{f}_{\mathrm{act}} \geq \mathrm{f}_{\mathrm{avg}}$

(c) : if $q_{\text {act }} \geq q_{\text {min }}$ and $p_{\text {act }} \geq p_{\text {avg }}$ and $f_{\text {act }}<f_{\text {avg }}$

(d) : if $\mathrm{q}_{\mathrm{act}} \geq \mathrm{q}_{\min }$ and $\mathrm{p}_{\mathrm{act}} \leq \mathrm{p}_{\text {avg }}$ and $\mathrm{f}_{\mathrm{act}} \leq \mathrm{f}_{\mathrm{avg}}$

(e) : if $\mathrm{q}_{\mathrm{act}}<\mathrm{q}_{\mathrm{min}}$

, in which $\mathrm{q}_{\text {act }}$ is the actual quality of the product delivered by seller $s, \mathrm{q}_{\exp }$ is the desired expected quality and $\mathrm{q}_{\min }$ is the minimum quality expected by $b$. pact is the price paid by $b$ to purchase the product from seller $s$. $p_{\min }$ is the minimum price quote, $p_{\max }$ is the maximum price quote received and $p_{a v g}$ is the average of the price quotes received by the buyer for this product.

The evaluator function should be proportional to the degree the quality delivered by the seller meets the buyer's expectations and the price paid to purchase the product and adjacency of buyers and salesrooms. If there are two sellers, s1 and s2, who can meet the buyer's expectation for the quality of the product, and s1's price, is lower than s2, and s1's distance (between $s 1$ and referent seller) is less than $s 2$ then $s 1$ should get a higher rating than s2. We make the common assumption that it costs more to produce a higher quality product. So when considering the price charged by a seller, if the seller meets the buyer's minimum expectation for quality, and if the price is greater than the average price quoted, and distance is more than $f_{\text {avg }}$, then the difference between the seller's price and the average price quoted and difference between the seller's distance and the $f_{\text {avg }}$ are weighed proportional against the average price quoted (average distance) for that product (part (a) of the equation 1). On the other hand, if the price of the seller is below the average price and distance is less than average distance (which can happen if the buyer is close to central market (where purchase occurs) and the sellers are competing to have more customers or there are too many low quality sellers) then the rating for this seller is computed based on its quality alone (part (d) of the equation 1). If the seller's quality does not meet the buyer's expectation then the difference of seller's price and the minimum price quoted and difference between actual distance and minimum distance is compared to the difference between the maximum and the minimum price (distance) quoted to penalize the seller more severely (part (e) of the equation).

In equation $1, \mathrm{f}_{\text {act }}$ is the distance between buyer and market place. $f_{\min }, f_{\max }$ is the minimum, maximum distance between buyer and seller; respectively, and $\mathrm{f}_{\mathrm{avg}}$ is the average distance between buyer and seller positions.

Computation of $\mathrm{f}_{\min }, \mathrm{f}_{\max }$ is a complicated problem. These variants depend on buyer population and salesrooms scattering in urban environment, number of market places and partly urban environment ambit. In this paper we don't intend to clarify how $f_{\min }, f_{\max }$ are calculated. coef $f_{\text {rep }}$ and coef $_{\text {far }}$ are coefficients, with values in the interval $[1,1.4]$, to emphasize on trust or distance, respectively.

This model makes the assumption that buyer $b$ expects the highest quality and nearest seller in the best case $\mathrm{q}_{\text {act }}$ can be equal to $\mathrm{q}_{\text {exp }}$ and it costs more to produce higher quality products. From the above equation it can be seen that $\mathrm{D}_{\mathrm{b}}{ }^{\mathrm{s}}$ ranges from $[-1,1]$. In the best case, $b$ gets the expected quality at the lowest price from nearest seller it means $\mathrm{D}_{\mathrm{b}}{ }^{\mathrm{s}(\max )}=1$. In the worst case, $\mathrm{q}_{\mathrm{act}}=0$ and $b$ pays the maximum price quoted and $\mathrm{D}_{\mathrm{b}}{ }^{\mathrm{s}(\mathrm{min})}=-1$. If the buyer has not interacted with the seller then $\mathrm{D}_{\mathrm{b}}{ }^{\mathrm{s}}=0$ for that seller and such a seller is referred to as a new seller.

Whenever buyer $b$ is evaluating a list of sellers for purchase decisions he/she computes $\mathrm{D}_{\mathrm{b}}{ }^{\mathrm{s}(\mathrm{avg})}$, the 
average rating for each seller $s$ from its past interactions. We define decision function $\mathrm{D}_{\mathrm{b}}^{\mathrm{s}(\mathrm{avg})}$ according to equation 2 .

$\mathrm{D}_{\mathrm{b}}^{\mathrm{s}(\mathrm{avg})}$ is computed as the weighted mean of the past $\mathrm{n}$ recent interactions (equations 3,4 ).

$$
D_{b}^{s(a v g)}=\frac{1}{W} \sum_{i=1}^{n} w_{i} D_{b(i)}^{s}
$$

where

$$
\begin{aligned}
& w_{i}=\frac{t_{c u r}}{t_{c u r}-t_{i}} \\
& W=\sum_{i=1}^{n} w_{i}
\end{aligned}
$$

Where $\mathrm{D}_{\mathrm{b}}{ }^{\mathrm{s}}$ is the rating computed for a direct interaction using equation 1. Subscript $i$, in parenthesis, indicate the $i$ 'th interaction. $w_{i}$ is the importance of the rating in computing the average. Recent ratings should have more importance. Hence the weight of a rating is inversely proportional to the difference between the times transaction happened $t_{i}$ to the current time $t_{\text {cur }}$.

The buyers have threshold values $\omega_{1}$ and $\omega_{2}$ for the decision function $\mathrm{D}_{\mathrm{b}}{ }^{\mathrm{s}(\mathrm{avg})}$ to indicate their satisfaction or dissatisfaction with the sellers, respectively. $\omega_{1}$ and $\omega_{2}$ are set by the buyers and $\omega_{1}>\omega_{2}$ and $\omega_{1}$ and $\omega_{2}$ are in the range $[-1,1]$. Buyers choose sellers whose average value of the decision functions are greater than or equal to $\omega 1$ and consider sellers to be reputable and accessible. Buyers do not choose sellers whose average decision functions are less than or equal to $\omega 2$ and consider them to be disreputable and far. It is unsure or far for sellers whose average decision functions ratings are between $\omega_{1}$ and $\omega_{2}$ and will consider them again only if there are no reputable/near or new sellers to consider. From the list of sellers who have submitted price bids, reputable/near sellers whose $\mathrm{D}_{\mathrm{b}}{ }^{\mathrm{s}(\mathrm{avg})}$ is above the satisfaction threshold $\omega_{1}$ are identified as potential sellers. Buyers include new sellers into the list of potential sellers to be able to quickly identify a good seller. For a seller with whom the buyers have interacted before, the quality is the average of the quality delivered in the past interactions. For a seller with whom the buyers have not interacted directly, the quality is set to the expected quality. From the list of potential sellers, buyers choose a seller randomly (or the seller who maximizes their product valuation functions). Product evaluation function is a function of the price a seller is currently quoting and the quality that has been delivered in the past and distance between the seller and the buyer.

\section{CASE STUDY}

Now, we describe a scenario and investigate its results. In this scenario we developed a multi-agent based geo-simulation of a market with autonomous buying and selling agents. When buyers want to purchase their products, they obtain registered lists of sellers selling the products and sends a message to each of the sellers in the list to submit their bids for the product $g$. Sellers who are interested in getting the contract submit bids which includes the price. The buyers wait for a certain amount of time for responses and then evaluate the bids received to choose a seller to purchase from. The following parameters were set. Quality $q$ sold across the sellers $(20 \leq \mathrm{q} \leq 50)$. Buyers expect a minimum quality of 40 $\left(\mathrm{q}_{\min }=40\right)$ and minimum distance (fact).

The price of a product for an honest seller is $\mathrm{pr}=\mathrm{q} \pm$ $10 \%$ q. We make the assumption that it costs more to produce high quality goods. We also make the reasonable assumption that the seller may offer a discount to attract the buyers in the market or raise its price slightly to increase its profits [10]. Hence the price of the product is set to be in the range $90 \%$ $110 \%$ of the quality for an honest seller. A dishonest seller, on the other hand, may charge higher prices. The distance between buyer and shop is calculated too. The sellers' evaluation of their profit is a function of the buying number per time unit. Buying number of every buyer is a function of his income (income level). Each time buyers purchase products from sellers their products evaluation are computed and we consider this as the buyers' gain for having purchased from that seller.

Majority of the buyers that are used in this experiment are F\&NF (Frequent and Infrequent). It is assumed that 10 to 15 percent of buyers do random shopping. Expected quality is $\mathrm{q}_{\mathrm{exp}}=50 ; 40 \leq$ acceptable-quality $\leq 50 ; 10 \leq$ nonacceptable-quality $\leq$ $39 ; \mathrm{p}_{\max }=55 ; \mathrm{p}_{\min }=18 ; \mathrm{p}_{\mathrm{avg}}=37.5 ; \mathrm{f}_{\max }=40 ; \mathrm{f}_{\min }=0$; $f_{a v g}=22$. High threshold value, $\omega_{1}$, and Low threshold value, $\omega_{2}$, can be computed as follows:

The buyer is expecting at least a quality of 40 . In the worst case it can get this at the highest price that can be charged by an honest seller which would be 44

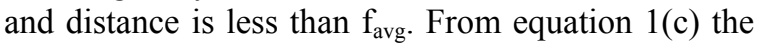
decision rating for that seller would be:

$$
\frac{40}{50}-\frac{0.9 * 44-0.9 * 37.5}{55-18}=0.641
$$

If the buyer expects at least quality of 40 , in the worst case he/she can get this at the most farness that can 
be 44 . In this case $\left(\mathrm{p}_{\text {act }}<\mathrm{p}_{\text {avg }}, \mathrm{f}_{\text {act }}=\mathrm{f}_{\text {avg }}\right)$ we calculate the decision rating from equation $1(\mathrm{~b})$.

$$
\frac{40}{50}-\frac{(2.2649-1.9354) 22}{44-0}=0.635
$$

So, we set $\omega_{1}=0.635$. For new sellers the decision function is set to 0 . These buyers should not come under the category of improper sellers. So we set the threshold value for a seller to be considered unacceptable as -0.1 . So $\omega_{2}=-0.1$

Applying random shopping in our scenario; 10 to 15 percent of shopping times, buyers buy randomly. We populated the market with 4 sellers; all of them belonging to one category (from the six categories). We can choose sellers from different categories. Sellers' price and quality properties are shown as:

1. Honest Acceptable (HA): each seller offers a quality in the range [40-50]. Price is between 90$110 \%$ of the quality they are selling.

2. Honest Not Acceptable (HNA): each seller offers a quality in the range [20-39]. Their price is between $90-110 \%$ of the quality they are selling.

3. Overpriced Acceptable (OPA): each seller offers a quality in the range [40-50]. Price is between 111$200 \%$ of the quality they are selling.

4. Overpriced Not Acceptable (OPNA): each seller offers a quality in the range [20-39]. Their price is between $111-200 \%$ of the quality they are selling.

5. Inconsistent: each seller offers a quality in the range [20-50]. Price is between $90-110 \%$ of the quality they are selling.

6. Dishonest: this category of sellers in their first sale to a buyer offer acceptable quality q [40-50] charging a price $\mathrm{pr}=\mathrm{q} \pm 10 \% \mathrm{q}$. In their subsequent sales to that buyer they reduce the quality $\mathrm{q}$ to be in the range [2030]. However their price still remains high. Price pr= $\mathrm{q} 1 \pm 10 \% \mathrm{q} 1$ where $\mathrm{q} 1$ is in the range $[40-50]$.

The places of sellers are chosen by users and data from the experiments was collected with 1000 buyer agent. In each simulation, buying agents conducted uncertain number of transactions. In each transaction they purchased some products (depend on their incomes) by querying the seller list and obtain price quotes from different sellers and utilize their buying strategy to choose a seller. We compared the performances of the sellers (in the form of number of transactions per time unit) to chose the best location of sellers. Figure 1 shows simulation environment consist of buyers and sellers.

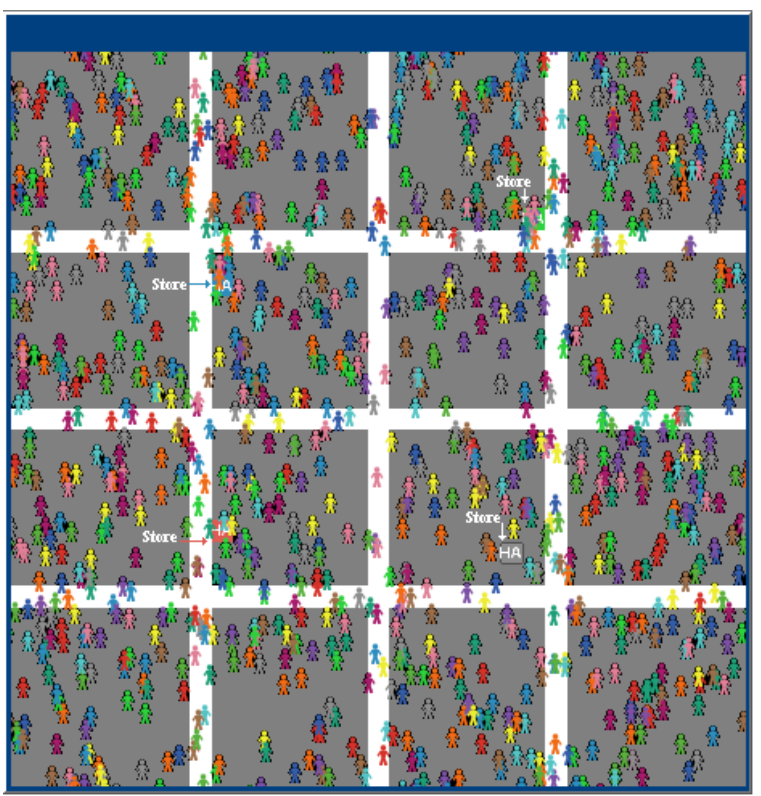

Figure 1. Simulation Environment (Buyers, Sellers and ...)

All sellers are HA. The simulation can be done around of resulted point to obtain accurate place.

Figure 2 shows simulation results that consist of transaction rate for four sellers. This survey reveals that seller 3 has the maximum transaction number. We can do simulation around of seller 3 to acquire accurate location.

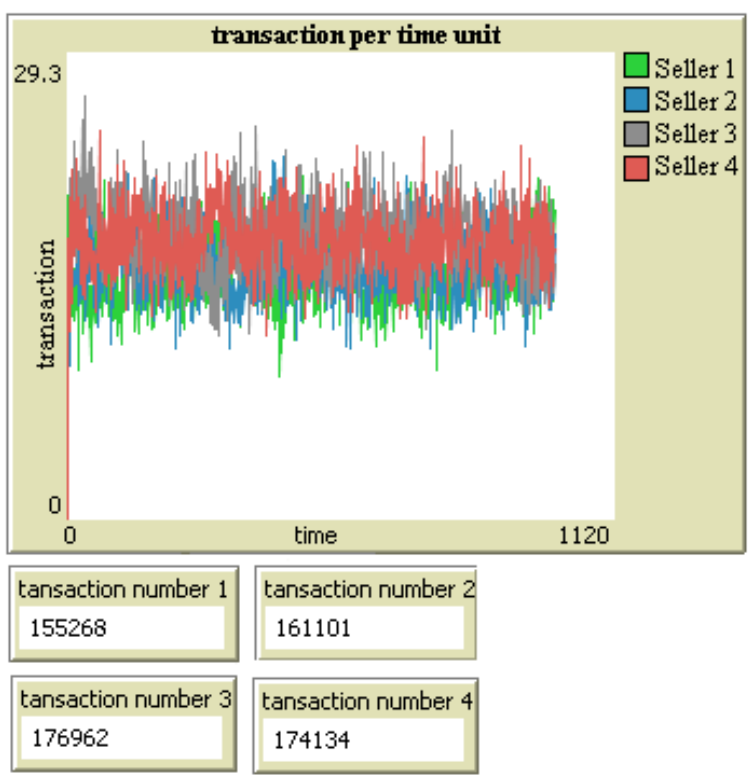

Figure 2. Transaction Rate and Transaction Number For Every Seller 


\section{CONCLUSIONS}

In this paper, an agent based model presented for buyer-seller interactions, sellers' location and category to be chosen by user. Buyers investigate the sellers' acting and a strategy to choose sellers in decentralized, open, dynamic, uncertain and untrusted multi-agent based markets. The buyer agents evaluate the seller agent's reputation and accessibility based on its ability to meet their expectations of product, service, quality, adjacency and price as compared to its competitors. According to specified sellers' location and buyer's strategy, the best location for seller is determined.

\section{REFERENCES}

1. Andreas, Koch. (2000). Linking Multi Agent Systems And GIS - Modeling And Simulating Spatial InterActions. Available: www.rwthaachen.de/geo/Ww/deutsch/MultiAgentsKoch.P DF

2. Moulin, Bernard., Walid, Ali. (22-SEP-06). Perception-Based Multi-Agent Geo-Simulation in the service of Retail Location DecisionMaking in a Shopping Mall. Canadian Journal of Regional Science.

3. Jiang, B. (10-12 August 2000). Agent-Based Approach To Modelling Environmental And Urban Systems Within GIS. Proceedings of 9th International Symposium on Spatial Data Handling, P.R.China

4. António S, Gonçalves Armanda Rodrigues Luís Correia. (2004). Multi-Agent Simulation Within Geographic Information Systems. Proceedings of 5th Workshop on Agent-Based Simulation

5. Cláudio Antônio da Silva., Rua Marquês de São Vicente., Roberto de Beauclair Seixas. (2005). Geographical Information Systems and Dynamic Modelling via Agent Based Systems. Available: www.impa.br/ rbs/pdf/acmgis_2005.pdf.

6. Roozmand, Omid., Ghasem Aghaee, Naser. (2007). Applying K-nn and Reforcement Learning for Buyer and Seller Agents. Proceeding of 12'th International Conference of Iran Computer Society

7. Walid, Ali., Moulin, Bernard., "Developing 2D3D Agent Based Simulations In Geographic Environment: An Approach And its Application to Simulate Shopping Behavior A Mall”, 2005.

8. Christian, J., E, Castle. (Sep 06). Principles and Concepts of Agent-Based Modelling for Developing Geospatial Simulations.

9. Angers. (2007). Reputation Based Buyer Strategy For Seller Selection For Both Frequent and Infrequent Purchases. Proceeding of 4th
International Conference on Informatics in Control, Automation \& Robotics

10. Bazghandi, Ali., Pouyan, Ali. (2008) Considering Geographic Information Systems In Buyer-Seller Agents Simulation. Damascus, Syria. Proceeding of 3'th Int. Conf (ICTTA'08)

11. Longley, P.A. and Batty, M. (2003), 'Prologue: Advanced Spatial Analysis: Extending GIS', in Longley, P.A. and Batty, M. (eds.), Advanced Spatial Analysis: The CASA Book of GIS, ERSI Press, Redlands, California

12. Bonabeau, E. (2002), 'Agent-Based Modelling: Methods and Techniques for Simulating Human Systems', Proceedings of the National Academy of Sciences of the United States of America (PNAS), 99(3): 7280-7287.

13. Russell, S. and Norvig, P. (2003), Artificial Intelligence: A Modern Approach, Prentice Hall, USA.

14. Brown, D.G. (2006), 'Agent-Based Models', in Geist, H. (ed.) The Earth's Changing Land: An Encyclopedia of Land-Use and Land-Cover Change, Greenwood Publishing Group, Westport, pp. 7-13.

15. Wegener, M. (2000), 'Spatial Models and GIS', in Fotheringham, A.S. and Wegener, M. (eds.), Spatial Models and GIS: New Potential and New Methods, Taylor and Francis, London.

16. Goodchild, M.F. (2005), 'GIS, Spatial Analysis, and Modelling Overview', in Maguire, D.J., Batty, M. and Goodchild M, F. (eds.), GIS, Spatial Analysis and Modelling, ESRI Press, Redlands, California. 